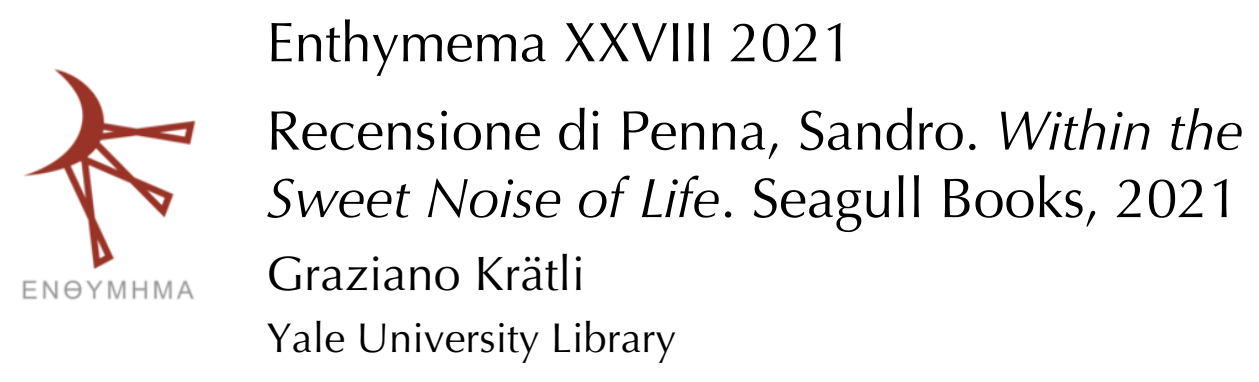

Abstract - Recensione di Penna, Sandro. Within the Sweet Noise of Life. Seagull Books, 2021.

Parole chiave - Poesia italiana; Sandro Penna; Traduzioni in inglese; Letteratura mondiale.

Abstract - Review of Penna, Sandro. Within the Sweet Noise of Life. Seagull Books, 2021.

Keywords - Italian Poetry; Sandro Penna; English Translations; World Literature.

Krätli, Graziano. "Recensione di Penna, Sandro. Within the Sweet Noise of Life. Seagull Books, 2021". Enthymema, n. XXVIII, 2021, pp. 251-254.

http://dx.doi.org/10.54103/2037-2426/16616

https://riviste.unimi.it/index.php/enthymema

(c) (i) Creative Commons Attribution 4.0 Unported License

ISSN 2037-2426 


\title{
Recensione di Penna, Sandro. Within the Sweet Noise of Life. Seagull Books, 2021
}

\author{
Graziano Krätli \\ Yale University Library
}

In italiano, il termine letteratura mondiale sembra mettere in evidenza, anche più del suo equivalente inglese World Literature (similmente un calco del tedesco Weltliteratur), la "straordinaria elusività» del concetto di universalità della poesia (Damrosch 1), che, secondo la formulazione originaria di Goethe, si manifesterebbe nella facoltà di rivelarsi ovunque e in qualsiasi epoca a centinaia e centinaia di uomini. Il carattere di tale "rivelazione" è oscuro e probabilmente impossibile da definire, ma non le sue condizioni materiali, che fanno affidamento sulla traduzione, seguita dalla produzione e circolazione di testi (manoscritti, a stampa o elettronici) per mezzo di libri, periodici o altri documenti, sia a stampa che digitali. La traduzione, letteraria o meno, è attività transculturale per eccellenza, le cui origini risalgono a quelle del linguaggio umano stesso. In quanto alla produzione e circolazione di manoscritti, la domanda e l'offerta di materiali (pellami, carte, inchiostri, ecc.), utensili, tecnologie e competenze è stata a lungo soddisfatta tramite reti commerciali e di comunicazione tese tra l'Europa, l'Africa e l'Asia. L'introduzione e la diffusione della stampa a caratteri mobili prima, e la rivoluzione digitale poi, hanno sfruttato tali reti modificandole, ampliandole, rafforzandole e trasformandole in un sistema distribuito per cui oggi la creazione, la circolazione e il consumo di contenuto, in qualsiasi forma e formato (tradizionale o elettronico), avvengono principalmente tramite pratiche e processi decentrati e dematerializzati, come dimostra il carattere transnazionale di gran parte dell'editoria, anche piccola e indipendente, e del lavoro culturale in genere.

Within the Sweet Noise of Life, a prescindere dal suo valore letterario (e dalla sua presenza materiale), è un caso rappresentativo di questo fenomeno. La sua esistenza è dovuta a un cast internazionale che comprende, in ordine di apparizione, un poeta italiano del Novecento, un traduttore americano che ha vissuto a Roma e attualmente risiede a Berlino e un editore indiano di ampio respiro culturale. A questi ruoli principali (di "autore", "regista" e "produttore") possiamo aggiungere l'équipe tecnica, segnatamente la composizione digitale a Calcutta, la stampa e la rilegatura a East Peoria, nell'Illinois, la distribuzione internazionale a Chicago e una rappresentanza commerciale a Londra. Parimenti internazionali saranno la ricezione critica, a cominciare dalla presente recensione (in italiano su una rivista italiana, ma redatta da un autore che risiede negli Stati Uniti e si occupa principalmente di letteratura indiana anglofona), cui se ne aggiungeranno forse altre, non solo nei paesi in cui il libro è distribuito, ma anche in Italia, dove ha le sue origini genealogiche.

Tali origini si riferiscono ovviamente al contenuto del libro, mentre il suo carattere merceologico e culturale, vale a dire il contenitore, è dovuto all'editore, Naveen Kishore, la cui intraprendenza illuminata è stata riconosciuta di recente con l'Ottaway Award for the Promotion of International Literature. La Seagull Books ha esordito nel 1982 con un catalogo incentrato sulla nuova drammaturgia indiana (e con traduzioni inglesi di opere in lingue regionali), che è poi cresciuto sia quantitativamente che geograficamente, tanto che oggi include opere di ogni genere letterario e di autori africani, arabi, austriaci, francesi, indiani, italiani, norvegesi, slovacchi, svizzeri, tedeschi e ungheresi. Il periodo privilegiato è il Novecento, l'enfasi modernista, l'afflato marxista. La collana italiana include più di una ventina di titoli di filosofia (Agamben, 


\section{Recensione di Penna, Sandro. Within the Sweet Noise of Life \\ Graziano Krätli}

Jesi, Virno), storia (Balestrini, Ginzburg, Pintor), critica letteraria (Asor Rosa, Belpoliti, Fortini), narrativa (Pecoraro, Volponi) e poesia (Morante, Penna).

Il rapporto di Sandro Penna con l'India (rapporto puramente editoriale e postumo) ha un precedente in Confused Dream (1988), una scelta di poesie tradotte da George Scrivani per Hanuman Books, piccola casa editrice d'avanguardia fondata da Raymond Foye e Francesco Clemente, con sede editoriale al Chelsea Hotel di New York e produzione in India, dove Scrivani, dalla Kalakshetra Press di Madras (Chennai), sovrintendeva alla stampa e rilegatura dei libri che venivano poi distribuiti in America: un altro esempio di editoria indipendente a carattere transnazionale (oltre che transculturale). Dal punto di vista materiale, ossia del libro come oggetto e prodotto commerciale, la differenza tra Confused Dream (formato lillipuziano, brossura cucita artigianale, copertina in cartoncino monocromatico arancione) e Within the Sweet Noise of Life (formato $22 \times 13 \mathrm{~cm}$, particolarmente appropriato al testo poetico, copertina cartonata e sovraccoperta illustrata con una fotografia di von Gloeden) testimonia il progresso compiuto dall'editoria indiana negli ultimi trent'anni, come anche le sue ambizioni culturali e commerciali crescenti, e la sua competitività sul piano internazionale.

Confused Dream rappresenta un caso sui generis, sia per il formato (e le sue conseguenze tipografiche) sia per la selezione (apparentemente basata su tre volumi, di cui però solo due identificati chiaramente), $\mathrm{ma}$ ha il pregio di essere una delle prime edizioni di poesie di Penna tradotte in inglese. A precederla furono quelle di Ian Young e Anthony Reid (1975), un canadese e un inglese, entrambi antologisti di letteratura omosessuale, e del poeta americano W.S. Di Piero (1982). La prima, pubblicata quando il poeta era ancora in vita, non figura nella bibliografia dell'edizione Meridiani Mondadori (Penna, Poesie, prose e diari 1362) ed è oggi introvabile. La seconda, cominciata un decennio prima della data di pubblicazione, è rimasta la più autorevole fino all'edizione di Alexander Booth, che la segue spesso (Di Piero è l'unico precursore menzionato nei ringraziamenti) e ancor più spesso ne supera il modello. Meno convincenti, anche se non del tutto trascurabili, quelle successive di William Jay Smith (1989) per un numero della rivista Poetry dedicato alla poesia italiana del secondo dopoguerra, Blake Robinson (1993) e Peter Valente (2014).

Per un traduttore, il confronto con i propri precursori è inevitabile quanto necessario; infatti, tanto inevitabile e necessario quanto il rapporto con l'originale, che in questo confronto risulta mediato, ossia chiarito e offuscato al tempo stesso, dalle versioni precedenti. Tale conversazione a più voci, in cui il traduttore presta ascolto simultaneamente all'autore e ai suoi interpreti (o, meglio, all'autore attraverso i suoi interpreti), può essere estenuante ma ha il vantaggio di fornire modelli, soluzioni ed esempi, offrendo al contempo la possibilità di operare una selezione critica nei loro confronti: scartando, accogliendo e adattando. (È necessario aggiungere che tale processo di adattamento selettivo avviene comunque, sia che il traduttore consulti eventuali precursori sia che operi "in solitaria").

Con Within the Sweet Noise of Life, Booth offre un saggio lodevole di come il confronto di cui sopra possa produrre non solo traduzioni fedeli, ma poesia in grado di essere percepita e apprezzata come tale nella lingua di arrivo.

Come altre precedenti, la selezione si apre con "La vita... è ricordarsi di un risveglio", la poesia «che Penna ha sempre voluto ad apertura del proprio canzoniere» (Penna, Poesie, prose e diari 1016), considerandola la sua prima (745), e che Booth rende adottando, scartando e adattando le scelte dei suoi precursori. «Waking sad» (per «risveglio triste»), «broken body» (per «corpo rotto»), «sudden release» (per «liberazione improvvisa») e «A crisp and colourful sea» (per «un mare tutto fresco di colore») provengono da Di Piero, mentre «uncertain light» (per «luce incerta») è la soluzione adottata da Smith (Di Piero traduce «tentative» e Scrivani «dim»). Booth inoltre corregge $i$ tempi verbali della prima strofe («to remember waking... / having seen... / having felt»), rendendoli più aderenti all'originale, dal quale sia Di Piero che Smith, e soprattutto Scrivani, si erano allontanati. Ma a differenza di tutti e tre (e dello stesso Penna), 


\section{Recensione di Penna, Sandro. Within the Sweet Noise of Life \\ Graziano Krätli}

invece di ripetere «outside» nella chiusa preferisce «beyond», una divergenza enfatica che pone in risalto il fatto che lo sguardo e l'attenzione del poeta, non solo passano dall'interno all'esterno («outside») dello scompartimento, ma, così facendo, passano oltre («beyond») la figura del giovane marinaio, incontrando un mare il cui colore, "tutto fresco", ricorda l'azzurro e il bianco della sua uniforme. Il risultato è più scorrevole nonché più fedele all'originale dei suoi tre predecessori.

Un altro esempio eloquente è "Guardando un ragazzo dormire". Scrivani, costretto dal formato della pagina, forza il testo in una struttura che ne stravolge il ritmo (e dunque il senso), allontanandosi dall'originale e impantanandosi in derive interpretative che culminano nell'assurdità del distico finale. Robinson, il cui titolo traduce più fedelmente quello di Penna, rende «in riva al mare» con «oceanside» (termine che fa pensare a una proprietà immobiliare) e «dormiranno» con «will go to sleep» (letteralmente andranno a dormire). Booth riduce l'età del soggetto, sia nel titolo («Watching a Young Boy Sleep») che nella traduzione di «fanciullo» con «little one», ed elimina la punteggiatura (una scelta che compie spesso e inspiegabilmente, soprattutto se si considera la generale fedeltà all'italiano di Penna), ma produce un risultato che, a differenza dei due precedenti, non suscita il desiderio (o la necessità) di consultare l'originale.

Nessuno dei tre traduttori, tuttavia, sembra riconoscere la profonda ambiguità (nel senso inteso da William Empson) che anima questa poesia, a cominciare dal primo verso e poi soprattutto nell'uso, ripetuto, dell'avverbio «ancóra». Penna infatti scrive «Tu morirai fanciullo ed io ugualmente» e non «Tu morirai, fanciullo, ed io ugualmente», lasciando aperta la possibilità che il fanciullo non sia il ragazzo del titolo ma la condizione (la fanciullinità, o una variante dell'eterno femminino) in cui sia lui che il poeta sono destinati sub specie aeternitatis. Similmente, i due versi successivi («Ma più belli di te ragazzi ancora / Dormiranno nel sole in riva al mare»), possono essere letti «Ma ragazzi ancora più belli di te / Dormiranno nel sole in riva al mare» (questo il senso attribuito dai traduttori) oppure «Ma ragazzi più belli di te / Dormiranno ancora nel sole in riva al mare». In quanto al verso finale («Ma non saremo che noi stessi ancora»), Booth è l'unico ad esprimerne il senso attraverso l'uso del verso al negativo («And yet, we'll be nothing but ourselves again»).

La poesia italiana del Novecento presenta alcuni casi limite di intraducibilità, soprattutto in componimenti (si pensi a "M'illumino d'immenso" o "Ed è subito sera") che per concisione ed ermetismo costringono a scelte limitate, spesso forzate e quasi sempre insoddisfacenti. Benché non ci sia nulla di ermetico in «Io vivere vorrei addormentato / entro il dolce rumore della vita», un candido, fanciullesco manifesto personale considerato «il distico più celebre di Penna, il più citato dai lettori ma anche il più odiato dal suo autore» (Penna, Poesie, prose e diari 1132), esso rappresenta uno di questi casi, motivo per cui è stato spesso evitato dai traduttori. Di Piero lo traduce pedissequamente «I'd like to live falling to sleep / inside all of life's sweet noise», rendendo «addormentato» con «falling to sleep» (variante di falling asleep, che descrive l'atto di addormentarsi anziché la condizione di essere addormentato) e aggiungendo «all» per ragioni metriche (cosicché, ritradotta, la poesia sarebbe «Io vorrei vivere addormentandomi / entro tutto il rumore della vita»). Booth, al contrario, compie un'operazione interpreativa che risulta in una versione elegante, originale e convincente: «Lullabied I'd like to live / Within the sweet noise of life». Dove «lullabied» trae ispirazione e giustificazione dal «dolce rumore della vita», che agisce come ninnananna e anche culla o grembo materno («entro»/ «within»).

In conclusione, al contrario di molte traduzioni che mettono in evidenza, passivamente o programmaticamente, l'intraducibilità di un poeta, Within the Sweet Noise of Life ha il pregio di affermare non solo la traducibilità di Penna, ma anche e soprattutto la sua universalità poetica (nel senso auspicato da Goethe), benché limitatamente al contesto anglofono. Un risultato notevole, oltre che difficilmente superabile. 


\section{Recensione di Penna, Sandro. Within the Sweet Noise of Life Graziano Krätli}

\section{Bibliografia}

Damrosch, David. What Is World Literature? Princeton UP, 2003.

Penna, Sandro. A Boy Asleep Under the Sun: Versions of Sandro Penna. Tradotto da Peter Valente, punctum books, 2014.

- Confused Dream. A cura di Raymond Foye \& Francesco Clemente, tradotto da George Scrivani, Hanuman Books, 1988.

—. Poesie, prose e diari. A cura di Roberto Deidier, Mondadori 2017.

- Poetry 155, no. 1/2, Italian Poetry since World W ar II. Tradotto da William Jay Smith, ottobrenovembre, 1989, pp. 14-17.

—. Remember Me, God of Love. Tradotto da Blake Robinson, Carcanet, 1993.

—. Thirty Poems. A cura di Ian Young e Anthony Reid, Catalyst, 1975.

—. This Strange Joy: Selected Poems of Sandro Penna. Tradotto da W.S. Di Piero, Ohio State UP, 1982. 